\title{
Simultaneous Occurrence of Sydenham Chorea with Erythema Marginatum: A Case of Rheumatic Fever
}

This article was published in the following Dove Press journal:

Pediatric Health, Medicine and Therapeutics

\author{
Temesgen Tsega Desta' \\ Mulugeta Naizgi $\mathbb{D}^{2}$
}

'Saint Paul Hospital Millennium Medical College, Addis Ababa, Ethiopia; ${ }^{2}$ Ayder Hospital, Mekelle University, Tigray, Ethiopia
Correspondence: Temesgen Tsega Desta Tel +25I91I023103

Email yafettemu@gmail.com
Background: Rheumatic fever continues to be a major public health problem in the developing world, being responsible for many morbidities and mortalities. Were it not for its serious effects on the cardiovascular system, and to some extent on the central nervous system, the disease might not have significant consequences. The central nervous system involvement is explained with Sydenham chorea in which case the patient manifests with purposeless and choreiform movements aggravated by stress. Apart from this movement disorder the patient will also demonstrate emotional liability and motor manifestations. It is seen more commonly in children and young adolescent girls. It usually manifests as an isolated phenomenon called pure chorea, without evidence of active rheumatic fever; the other rare form of which is a type of acute rheumatic fever.

Clinical Description: This case report is about a nine-year-old female child presenting with two weeks of complaint of abnormal purposeless, non-rhythmic movement of extremities and the face, with failure to communicate. At presentation, she had non-pruritic skin lesions that had appeared a day prior to admission. The skin lesions were circular, red in color, and painless, involving the abdomen, and lower chest anteriorly, and extending to all extremities and the back. Two months previously she had a history of sore throat and treatment with unspecified P.O. medication. Antistreptolysin O (ASO) antibody was 430 Todd units $/ \mathrm{mL}$ and the erythrocyte sedimentation rate was $58 \mathrm{~mm} / \mathrm{h}$. Echocardiography study revealed carditis (no evidence of chronicity) with moderate to severe MR, mild TR, no pulmonary hypertension, good biventricular function, and no pericardial effusion.

Conclusion: In this case report, we would like to show the central nervous system manifestation of rheumatic fever called Sydenham chorea seen together with erythema marginatum and other features of rheumatic fever.

Keywords: Sydenham chorea, erythema marginatum, rheumatic recurrence, antistreptolysin O antibody

\section{Background}

Even though it has declined significantly in developed countries over the last decades, rheumatic fever continues to be a major public health problem in developing countries. Consistent with the above description the prevalence of rheumatic fever is high in Ethiopia. In a cross-sectional study that was conducted from April 1, 2013 to December 10, 2014 in 28 selected primary schools distributed across different regions, the nationwide prevalence of definite rheumatic heart disease among school children (between the age of 6 and 18) was found to be 14 cases per 1000. ${ }^{1}$

Rheumatic fever is a result of post-group A streptococcal infection, with its highest peak occurring between the ages of 5 and 15 years old. A preceding 
occurrence of tonsilopharyngitis by certain streptococcal serotypes can result in an abnormal immune response that will target organs of the body, including heart, joint, skin and brain, which have antigenic similarity with the group A streptococcal organism. Although the other manifestations are self-limiting and resolve without sequelae, carditis may result in chronic rheumatic heart disease (RHD) with associated significant morbidity and mortality from heart failure and related complications. ${ }^{2}$ Once the patient developed rheumatic fever there is a high probability for the patient to have another attack, which is referred to as rheumatic recurrence. The prognosis and natural history of rheumatic carditis and RHD are strongly influenced by both the severity of the initial carditis and RF recurrences. ${ }^{3}$

The World Health Organization has published guidelines in 2002-2003 for the diagnosis of rheumatic fever and rheumatic heart disease. In these guidelines, two separate diagnostic criteria were mentioned for the diagnosis of rheumatic recurrence for patients with and without established RHD. A recurrent attack of $\mathrm{RF}$ in a patient without $\mathrm{RHD}$ requires fulfillment of the Jones criteria (two major or one major with two minor criteria, plus evidence of a preceding streptococcal infection) and a recurrent attack of RF in a patient with underlying RHD requires two minor criteria and evidence of a preceding streptococcal infection. ${ }^{4}$ A recent modification in the Jones criteria in 2015 by the American Heart Association stated that the diagnostic criteria for a recurrent attack of RF in a patient with underlying RHD require two major or one major and two minor or three minor criteria and evidence of a preceding streptococcal infection. ${ }^{5}$

It is known that the clinical manifestations of Sydenham chorea occur due to neuropathologic changes and inflammation in the basal ganglia, cerebral cortex, and the cerebellum. Sydenham chorea occurs in $10 \%$ to $30 \%$ of the cases of $\mathrm{RF}^{2}$ Sydenham chorea tends to have a longer latency period after the sore throat infection compared to the other major clinical manifestations of acute rheumatic fever/recurrence. This may range from few months (1 to 6 months) to a year; therefore, it is uncommon for it to occur simultaneously with the other major clinical manifestations; acute phase reactants are often normal and antistreptococcal antibodies may not be elevated. When carditis and chorea are found in the same patient, it is often the chorea that prompts medical attention, at which time rheumatic cardiac involvement is detected. Although the combination of chorea and carditis is common, occurring in $47 \%$ of patients with RF in a recent series, ${ }^{6}$ the cardiac involvement tends to be mild and heart failure is uncommon. ${ }^{7}$

\section{Clinical Presentation}

A nine-year-old female referred to our hospital from the nearby primary hospital after she presented with two weeks of complaint of abnormal purposeless, non-rhythmic movement involving upper extremities and the face that improve during sleep. Associated with this she had an inability to speak since the onset of the complaint, but she can comprehend during communication. A day prior to presentation she started to have non-pruritic skin lesions which are circular, red in color and painless involving the abdomen, and lower chest anteriorly, that extend to all extremities and the back. Two months back she had a history of sore throat and treatment with unspecified medication (syrup - likely to be antibiotics). Recently she is not taking any medication and has no self or family history of known allergy. Her family and she denied any history of cardiac symptoms in the past which could suggest preexisting rheumatic heart disease.

Her physical examination revealed a choric movement in general appearance involving the upper extremities. There was no derangement in her vital signs except for low-grade fever. All of her anthropometric measurements were in the normal range. On cardiovascular precordial examination, the point of maximum impulse was at 6 th intercostal space at the left midclavicular line, systolic thrill at the apex, grade 3/6 holosystolic murmur (HSM) at the apex and grade 3/6 HSM at the Erb's point. In the musculoskeletal system, milk maid's grip, pronator drift, and darting sign were positive. During integumentary system examination by a consultant dermatologist on the day of admission there were circular skin lesions over the anterior trunk extending centrifugally, peripherally raised while clearing at the center and having a serpiginous pattern (Figure 1A-C). On the next day, similar lesions appeared in the proximal part of her extremities while the initial lesions on torso faded and subsequently disappeared.

Her sensory and motor systems were normal on central nervous system examination. She was investigated with complete blood count (CBC) having a result of white blood cell count (WBC) of 7100 (neutrophils 58.2\%, lymphocytes $26.4 \%$ ), hematocrit was $37.5 \%$, platelet count of 326,000 , the antistreptolysin O (ASO) antibody was 430 Todds units. Erythrocyte sedimentation rate was $58 \mathrm{~mm} / \mathrm{h}$, chest X-ray was normal while echocardiography study revealed carditis with moderate to severe MR, mild TR, no pulmonary hypertension, and good biventricular function with no pericardial effusion. The patient's electrocardiographic study revealed a normal finding. 
A

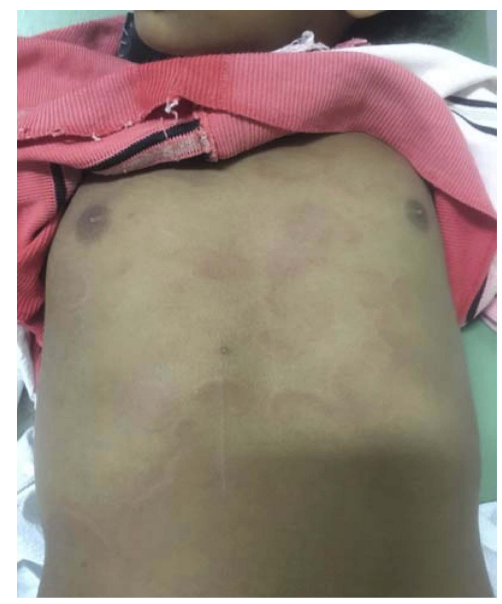

B

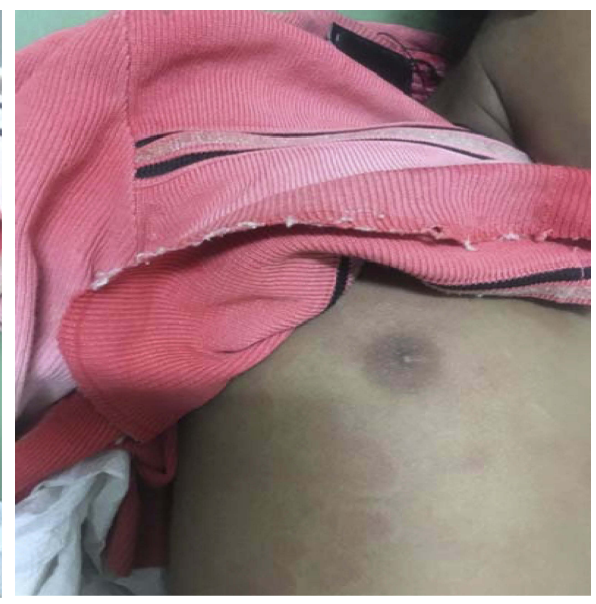

C

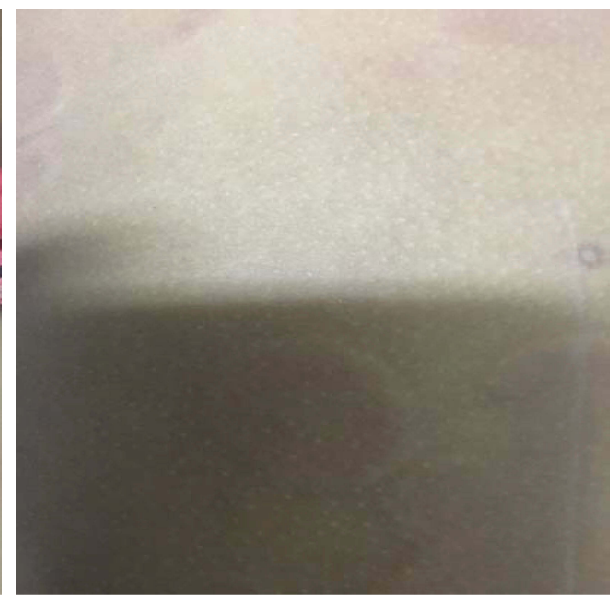

Figure I Circular raised peripheral erythematous lesion over the anterior trunk with central clearing. (A) Multiple annular skin lesions with erythmatous, raised border central LT clearing located at the anterior trunk. B and $\mathbf{C}$ are close-up views.

The final assessment was carditis (moderate to severe MR, mild TR), Sydenham chorea, and erythema marginatum.

After admission, she was given stat dose of benzathine penicillin intramuscularly and advised to continue thereafter on a monthly basis. Phenobarbitone $30 \mathrm{mg}$ orally to be taken three times a day and aspirin with a dose of $250 \mathrm{mg}$ oral four times in a day (QID) was initiated. With this management her condition improved with the skin lesion disappearing in three days and the choric movement decreasing after 5 days and she started to communicate without difficulty.

Later she got discharged after 10 days of stay in the hospital with complete resolution of the choric movement. She is appointed to return to the pediatric cardiac clinic after 14 days and advised to continue the above medications till then. During subsequent follow-ups, she continued with monthly benzathine penicillin and there was no complaint.

\section{Ethical Consideration}

This female child is nine years old and her legal guardian (her mother) who has been with her during admission and later in the follow-up gave consent for publication of this case report. The mother has been communicated with and provided with a consent form that is written in her local language, stating that she has no objection if this case is published and used for academic purposes; and pictures of the child can be used accordingly.

No institutional approval was needed for this case report publication.

\section{Conclusion}

This patient presented with a recent past history of sore throat followed with symptoms and signs suggestive of rheumatic fever together with supportive laboratory and echocardiographic evidence. The major complaint which brought this patient to our health facility was Sydenham chorea. In this case report, we would like to show this central nervous system manifestation of rheumatic fever seen together with erythema marginatum and other features of rheumatic fever. We could not find other published papers about a patient with similar presentations.

\section{Disclosure}

The authors report no conflicts of interest in this work.

\section{References}

1. Yadeta D, Hailu A, Haileamlak A, et al. Prevalence of rheumatic heart disease among school children in Ethiopia: A multisite echocardiography-based screening. Int J Cardiol. 2016;221:260-263. doi:10.1016/j. ijcard.2016.06.232

2. Allen HD, Driscoll DJ, Shaddy RE, Feltes TF. Moss and Adams' heart disease in infants, children, and adolescents: including the fetus and young adult. 8th edition. Philadelphia: Lippincott Williams \& Wilkins; 2013.

3. Bland EF, Jones D. Rheumatic fever and rheumatic heart disease: a twenty-year report on 1000 patients followed since childhood. Circulation. 1951;4:836-843. doi:10.1161/01.CIR .4 .6 .836

4. World Health Organization. Rheumatic fever and rheumatic heart disease. World Health Organ Tech Rep Ser. 2004;923:1-122. 
5. Gewitz MH, Baltimore RS, Tani LY, et al. Revision of the jones criteria for the diagnosis of acute rheumatic fever in the era of doppler echocardiography. Circulation. 2015;131:1806-1818. doi:10.1161/ CIR.0000000000000205

6. Veasy LG, Tani LY, Hill HR. Persistence of acute rheumatic fever in the intermountain area of the United States. J Pediatr. 1994;124:9-16. doi:10.1016/S0022-3476(94)70247-0
7. Feinstein AR, Spagnuolo M. The clinical patterns of acute rheumatic fever: a reappraisal. Medicine. 1962;41:279-305. doi:10.1097/00005792-1962 12000-00001

\section{Publish your work in this journal}

Pediatric Health, Medicine and Therapeutics is an international, peerreviewed, open access journal publishing original research, reports, editorials, reviews and commentaries. All aspects of health maintenance, preventative measures and disease treatment interventions are addressed within the journal. Practitioners from all disciplines are invited to submit their work as well as healthcare researchers and patient support groups. The manuscript management system is completely online and includes a very quick and fair peer-review system. Visit http://www.dovepress.com/testimonials.php to read real quotes from published authors.

Submit your manuscript here: http://www.dovepress.com/pediatric-health-medicine-and-therapeutics-journal 